Revue internationale P.M.E.

Économie et gestion de la petite et moyenne entreprise

\title{
L'implication à l'export des PME/PMI: le cas des sous-traitants de l'industrie aéronautique civile en Midi-Pyrénées
}

\section{François Thérin}

Volume 8, numéro 2, 1995

URI : https://id.erudit.org/iderudit/1008352ar

DOI : https://doi.org/10.7202/1008352ar

Aller au sommaire du numéro

Éditeur(s)

Presses de l’Université du Québec

ISSN

0776-5436 (imprimé)

1918-9699 (numérique)

Découvrir la revue

Citer cette note

Thérin, F. (1995). L'implication à l'export des PME/PMI: le cas des sous-traitants de l'industrie aéronautique civile en Midi-Pyrénées. Revue internationale P.M.E., 8(2), 119-146. https://doi.org/10.7202/1008352ar
Résumé de l'article

Cet article tente d'étudier les caractéristiques des PME /PMI exportatrices à travers leur performance économique et financière, leur politique d'innovation et leur diversification. L'étude a été menée auprès d'un échantillon de 48 PME/ PMI sous-traitantes de l'industrie aéronautique en Midi-Pyrénées. Les résultats montrent que les entreprises qui exportent le plus disposent d'une taille plus importante et d'une diversification en termes de secteurs clients plus faible que les non-exportateurs ou les petits exportateurs. 


\title{
Notes de recherche
}

\section{L'implication à l'export des PME/PMI: le cas des sous-traitants de l'industrie aéronautique civile en Midi-Pyrénées}

\author{
François THÉRIN* \\ École supérieure de commerce de Grenoble
}

MOTS CLÉS

\section{Exportation - PME / PMI - Sous-traitance - Aéronautique Approche quantitative - Données secondaires}

\begin{abstract}
RÉSUMÉ
Cet article tente d'étudier les caractéristiques des PME/PMI exportatrices à travers leur performance économique et financière, leur politique d'innovation et leur diversification. L'étude a été menée auprès d'un échantillon de 48 PME/ $P M I$ sous-traitantes de l'industrie aéronautique en Midi-Pyrénées. Les résultats montrent que les entreprises qui exportent le plus disposent d'une taille plus importante et d'une diversification en termes de secteurs clients plus faible que les non-exportateurs ou les petits exportateurs.
\end{abstract}

\begin{abstract}
This article studies the characteristics of small and medium sized export companies through their economic and financial performances, their innovation policies and their diversification. The study was carried out on 48 sample small and medium sized subcontractors in the aeronautics industry in Midi-Pyrénées.
\end{abstract}

* Après des études en management à l'École supérieure de commerce de Toulouse et un DEA à l'IAE d'Aix-en-Provence, l'auteur enseigne la stratégie et le management technologique au sein du Groupe ESC Grenoble. Il poursuit parallèlement un $\mathrm{Ph} . \mathrm{D}$. en management de la technologie à l'École polytechnique de Montréal. Ce texte a été écrit alors que l'auteur était à l'IAE d'Aix-en-Provence. Adresse : Groupe ESC Grenoble, 12, rue Pierre-Sémard, BP 127, 38003 Grenoble Cedex 01. L'auteur tient à remercier Élisabeth Lefebvre, professeure à l'École polytechnique de Montréal pour ses apports précieux en statistiques ainsi que les lecteurs anonymes pour leurs judicieux conseils. 
The results show that the companies which export most are larger in size but less diversified in terms of customer sectors than those companies who do not export at all or who export little.

\section{RESUMEN}

Este artículo intenta estudiar las caracteristicas de las PyMEs exportadoras a traves un dinamismo ecónomíco y financiero y también a traves la innovacíon y la diversificación. Este estudio se realizo a partir de una muestra de 48 PyMEs en el sector de la subcontratacion industrial aeronaútica en MidiPyrénées. Los resultados muestran que las empresas que exportan disponen de un tamanó más bajo que las que no exportan o las pequeñas empresas exportadoras.

\section{Introduction}

La sous-traitance aéronautique française affiche un chiffre d'affaires de 14,5 milliards de francs en 1990 et un effectif de 63000 employés, dont environ 16000 pour la région Midi-Pyrénées. Son activité est fortement dépendante de la stratégie de sous-traitance de l'Aérospatiale, le principal constructeur aéronautique français. Face à l'augmentation des plans de charge, les grands programmes font de plus en plus appel à la sous-traitance. Ainsi, le taux de sous-traitance de l'Aérospatiale est passé de $10 \%$ en 1974 à $40 \%$ de la charge pilotée en 1989. De plus, on assiste à une mise à niveau des sous-traitants : ils sont maintenant sévèrement sélectionnés; leur nombre est réduit et ils doivent être capables de produire des pièces vite et bien. La nouvelle démarche industrielle, mise en œuvre par les constructeurs, repose sur les principes fondamentaux suivants : un recentrage de l'activité des avionneurs sur leurs métiers spécifiques, un partage des risques et des financements en demandant aux sous-traitants majeurs de prendre à leur charge les frais d'études et de développement, l'acquisition des travaux sous-traités aux meilleurs coûts compatibles avec la qualité, ainsi que les délais et le développement d'un esprit de partenariat. Les sous-traitants doivent donc participer à la politique de réduction des coûts de production, lesquels avoisinent $20 \%$. Ces objectifs sont atteints par la mise en concurrence systématique des sous-traitants et par des appels d'offres nationaux, européens ou mondiaux qui placent ceux-ci dans une position permanente de recherche d'une meilleure productivité. Cette amélioration passe par de lourds investissements en moyens et en personnel qualifié, par la recherche d'une meilleure adéquation en matière d'assistance technique (normes-méthodes) ou commerciale et juridique (négociation, suivi des contrats). De même, la sectorisation mise en place (les sous-traitants de premier niveau pilotent une sous-traitance de deuxième ou troisième rang) permet de réduire les frais de gestion des grands donneurs d'ordre. L'Aérospatiale réduit le nombre de ses interlocuteurs de 400 à 100 , en substituant à la sous-traitance 
des suites d'opérations spécifiques auprès de plusieurs industriels en intégrant directement la fourniture d'une pièce terminée ou d'un sous-ensemble ou d'un ensemble complet sur les chaînes de montage.

L'instauration de ce nouveau mode de relations entre l'Aérospatiale et ses partenaires doit permettre de satisfaire un triple but: ne pas déstabiliser les potentiels régionaux, limiter le taux de dépendance au-dessous de $30 \%$ et permettre aux sous-traitants de se donner les moyens de leur subsistance en cas de baisse de la charge aéronautique. Cependant, les études réalisées par le Comité économique et social de la région Midi-Pyrénées en 1990 et l'OSTIAS ${ }^{1}$ en 1992 auprès des principaux sous-traitants mettent en lumière certains problèmes dont on peut distinguer le caractère structurel ou conjoncturel. Les problèmes structurels sont liés à la forte dépendance à l'égard des donneurs d'ordre. En effet, les sous-traitants hésitent à mener une politique d'investissement soutenue, car ils sont habitués au régime de charges cycliques spécifiques à cette industrie. De plus, les donneurs d'ordre tiennent à maintenir en interne les spécialités qu'ils trouvent chez leurs sous-traitants et favorisent donc plutôt la sous-traitance de capacité au détriment de la sous-traitance de spécialité. Ils diminuent actuellement le nombre de sous-traitants de premier niveau, et les informations en provenance des donneurs d'ordres sont souvent confuses et contradictoires, notamment en ce qui a trait aux prévisions de plan de charge, ce qui réduit encore la visibilité des sous-traitants. Pour ce qui est de la qualité, la mise en œuvre des politiques n'est pas très efficace. Alors qu'il suffit d'être agréé par un des membres du BDLI pour pouvoir travailler avec l'ensemble des donneurs d'ordre allemands, en France, les sous-traitants doivent satisfaire à des normes différentes pour les administrations, les organismes habilités (ISO, par exemple) et les principaux donneurs d'ordre (agréments spécifiques pour l'Aérospatiale, SNECMA et Dassault, par exemple). Pour assombrir le tableau, on peut y ajouter également une carence notable de personnel qualifié disponible. De plus, il existe deux principaux problèmes conjoncturels dont souffrent actuellement les sous-traitants. Des entreprises agréées sur le plan de la qualité se voient retirer des contrats au profit d'entreprises non agréées pouvant offrir des taux horaires inférieurs. De surcroît, la conséquence conjoncturelle du développement de la sous-traitance de capacité voulu par les donneurs d'ordre est un recours de plus en plus fréquent à des rapatriements d'activité, parfois sous forme de non-reconductions de contrats, qui permettent de juguler les crises internes, mais mettent gravement en cause la survie des sous-traitants.

Après avoir procédé à cet état des lieux, il convient de s'interroger sur les solutions possibles pour remédier à ces différents problèmes. Une étude

1. Observatoire de la sous-traitance de l'industrie aéronautique et spatiale. 
exhaustive des différentes possibilités dépasse le cadre de cette recherche. Cependant, parmi les solutions proposées pour améliorer la compétitivité des sous-traitants, l'extension géographique des marchés nous semble la plus intéressante à étudier car, comme l'ont souligné, entre autres, Kaynak et al. (1987), c'est une réponse possible à un marché saturé ou à une récession. De plus, elle peut s'adresser, pour ce secteur et dans cette région, à la fois aux sous-traitants de spécialité et de capacité, compte tenu de la présence d'une base industrielle en aéronautique solide, dans des pays européens accessibles du point de vue des distances (Espagne et Italie) et des démarches juridiques (en tant que membres de la CEE). L'exportation débute souvent dans des pays proches psychologiquement (Dichtl et al., 1984), et les barrières douanières sont également un obstacle à l'exportation.

\section{Revue de littérature et hypothèses de recherche}

\subsection{L'exportation des PME/PMI}

L'internationalisation des marchés a entraîné de nouveaux défis stratégiques pour les entreprises (Bartlett et Ghoshal, 1987a, 1987b). Celles-ci doivent subir les assauts d'entreprises souvent plus compétitives sur le plan coût/ qualité, en particulier celles des pays asiatiques. Heureusement pour les soustraitants français de l'industrie aéronautique, ils ont su conserver leur avantage historique en termes de maîtrise des savoir-faire particuliers de ce secteur. Cependant, ils doivent supporter le joug d'un client souvent unique. Les réponses naturelles à cette situation peuvent entraîner les entreprises vers la diversification. Pour certains auteurs (Calori et Harvatopoulos, 1988; Detrie, Mercier et Ramanantsoa, 1982), la diversification intervient, dans le développement de l'entreprise, après la pénétration du marché traditionnel et l'expansion géographique. Ces auteurs ne considèrent donc pas la diversification géographique comme une réelle diversification. Cependant, leur définition de la diversification nous indique qu'elle est soit défensive, pour remplacer ou réguler une activité de base défaillante ou irrégulière, soit offensive, pour profiter d'un avantage pour conquérir de nouvelles positions. En ce sens, il est donc possible de dire que les sous-traitants de l'aéronautique ont intérêt à développer une stratégie de diversification, car leur sort se trouve lié à une activité de base défaillante et irrégulière tout en possédant des avantages compétitifs certains en termes de savoir-faire. L'expansion géographique peut donc bien être qualifiée de diversification internationale.

Le problème de l'exportation fait partie des champs majeurs d'investigation en gestion et en économie, car il a des répercussions importantes sur l'activité économique d'un pays ou d'une région. Ainsi, pour la région Midi- 
Pyrénées, cadre de notre recherche, les exportations aéronautiques représentent, en 1992, 62\% des exportations de la région avec 33 milliards de francs (le deuxième poste, l'agriculture, ne représente que 4 milliards de francs) et dégagent un solde positif de 18 milliards de francs. Les facteurs favorisant ou inhibant les performances à l'export intéressent donc à la fois les gestionnaires et les responsables économiques.

De nombreuses études, depuis Tookey en 1964, ont tenté de caractériser certains facteurs affectant les capacités à l'export des entreprises. Les principales recherches se situent à deux niveaux : des recherches empiriques sur les facteurs explicatifs de l'exportation des PME/PMI et une tentative de définir les modèles du processus d'exportation (Olson et Wiedersheim-Paul, 1978 ; Wiedersheim-Paul et al., 1978; Aaby et Slater, 1989 ; Roux, 1991). Celles-ci se composent de caractéristiques externes (environnement de la firme) et internes (caractéristiques propres à l'entreprise, compétences et caractéristiques du dirigeant) qui influencent la stratégie de l'entreprise ou les perceptions du dirigeant, lesquelles vont alors conditionner soit la décision d'exporter, soit le niveau de ces exportations.

Cependant, la littérature concernant les études empiriques est jusqu'à présent inconsistante (Miesenbock, 1988; Aaby et Slater, 1989). Si l'on souhaite pouvoir dégager des critères communs concernant l'exportation, il nous semble logique d'étudier les dimensions les plus générales et les moins discutables d'une entreprise à l'autre. Les caractéristiques factuelles de l'entreprise, bien que peu étudiées dans la littérature, correspondent cependant à ces critères. Elles constitueront donc le centre d'intérêt de cette recherche. Nous avons préféré, s'agissant d'une première approche dans ce secteur, nous appuyer sur deux sources secondaires fiables et disposer de moins de liberté dans les variables étudiées. Parmi les différentes caractéristiques, nous avons donc retenu cinq dimensions peu analysées ou pour lesquelles le débat semble loin d'être clos: l'innovation, la performance économique et financière, la diversification, la productivité et la taille. Avec ces cinq caractéristiques, nous tenterons d'expliquer non pas la décision, mais l'implication à l'export, c'est-àdire le degré d'exportation des entreprises, qu'il soit nul, faible ou important.

\subsection{L'innovation}

Nous nous intéressons à la politique des entreprises en matière d'innovation. L'innovation est souvent étudiée par l'intermédiaire de la recherche et du développement (R-D) et en particulier la présence d'un service de R-D à l'intérieur de l'entreprise (Ong et Pearson, 1982). Cependant, ces études font le plus souvent référence au processus de diffusion de l'innovation et à la genèse du produit, c'est-à-dire à des aspects perceptifs de l'innovation, de son 
intégration dans les pratiques de l'entreprise, que l'on peut regrouper sous le terme d' «innovation organisationnelle» (Lefebvre et Lefebvre, 1992; Lefebvre et al., 1992; Lefebvre et al., 1991; Damanpour, 1991; McGee et Thomas, 1989). En étudiant la R-D dans les PME, nous partageons l'avis d'Acs et Audrestch (1990) qui va à l'encontre de la supposition répandue selon laquelle le changement technologique requiert des quantités de plus en plus grandes en R-D amassées et organisées principalement par de grandes entreprises (traduction libre).

Nous avons choisi de nous concentrer sur l'engagement de l'entreprise en recherche et développement. Les sous-traitants de l'aéronautique n'étant pas exclusivement des entreprises de production, mais aussi des bureaux d'études, il n'est pas possible de s'intéresser à l'introduction d'outils nouveaux, tels les machines à commandes numériques, la conception assistée par ordinateur (CAO) ou le juste-à-temps, ceux-ci n'ayant aucune signification pour certaines entreprises. Nous retiendrons donc comme variable d'innovation les frais de recherche et développement engagés par l'entreprise (Lefebvre et al., 1991 ; Ong et Pearson, 1982). Cependant, l'acquisition de savoir-faire pouvant se faire en interne (R-D), mais également en externe, nous retenons également les dépenses en achats de brevets et de concessions.

D'après la revue de littérature effectuée par Miesenbock (1988), les résultats concernant la R-D sont inconsistants: Burton et Schlegelmilch (1987), McGuiness et Little (1981) et Ogram (1982) ont montré que les exportateurs dépensaient plus d'argent en R-D que les entreprises qui n'exportent pas. Kirpalani et McIntosh (1980) ont établi une relation négative entre les ventes à l'export et les dépenses en R-D, alors que Schlegelmilch et Crook (1986) et Stening et McDougall (1975) (cités par Miesenbock, 1988) ont prouvé le contraire. Nous allons donc, dans le cas des PME/PMI soustraitantes de l'aéronautique, essayer de définir une relation, positive, négative ou nulle.

Première hypothèse: Il existe une relation entre l'implication à l'export des $P M E / P M I$ et l'innovation.

\subsection{La performance économique et financière}

Elle n'a été que peu étudiée dans la littérature et toujours reliée à la taille, selon le principe que la taille influence l'assise financière et la capacité à obtenir des fonds. Malekzadeh et Nahavandi (1985) se sont intéressés à l'avantage financier de la PME perçu par les dirigeants dans la compétition avec les autres firmes. Cependant, à notre connaissance, aucune étude ne porte sur l'influence directe de la performance économique et financière. Ce type de 
variables sera étudié dans la présente recherche en prenant comme hypothèse qu'elles ont une influence dans l'implication à l'export des PME / PMI.

Deuxième hypothèse: Il existe une relation entre la performance économique et financière et l'implication à l'export des PME/PMI.

\subsection{La diversification}

Devant un discours de plus en plus répandu appelant à la diversification des entreprises, l'étude de ce facteur est importante dans le cadre d'une performance à l'export. La diversification est scindée en deux types. Premièrement, plutôt que l'activité principale de l'entreprise, nous allons étudier les différents savoir-faire maîtrisés. Les savoir-faire se situent sur un plan moins général dans la mesure où une activité peut recouvrir plusieurs savoir-faire. À titre d'exemple, une entreprise ayant comme activité les constructions métalliques développera des savoir-faire en charpentes métalliques, en construction d'usines clé en main, en bureau d'étude et d'ingénierie. De même, la mécanique de précision pourra recouvrir la mécanique avec des machines à commande numérique, la robinetterie industrielle, la robotique et l'équipement pneumatique (ces deux exemples ont été pris parmi les entreprises de notre population). Nous préférerons donc employer ce critère, qui nous paraît plus explicite.

Si l'entreprise maîtrise plusieurs savoir-faire différents, cela peut jouer un rôle important dans l'exportation, en augmentant le nombre de marchés à conquérir. Cependant, cette diversification peut également constituer un handicap si elle est mal maîtrisée dans la mesure où l'entreprise ne dispose pas d'un réel savoir-faire, mais de plusieurs domaines de compétences plus ou moins intégrés.

Troisième hypothèse: Il existe une relation entre la diversification des savoir-faire et l'implication à l'export des PME/PMI.

Deuxièmement, nous étudierons la diversification en termes de secteurs clients. Là encore, le fait d'avoir de nombreux clients peut augmenter le nombre de débouchés à l'étranger ou, au contraire, diminuer la connaissance que l'entreprise aura de ses différents marchés et donc constituer un frein à l'exportation, le manque de connaissances des occasions d'affaires à l'étranger étant souvent cité (Czinkota et Johnston, 1983). Dans le secteur de la soustraitance aéronautique, les PME/PMI souffrent souvent de n'avoir qu'une seule entreprise cliente. Et cela est souvent dû au fait que leur client est présent uniquement dans le secteur aéronautique. Le fait de disposer de débouchés en dehors du secteur aéronautique permet donc de pallier les phénomènes conjoncturels et structurels de l'aéronautique et offre plus de débouchés à 
l'entreprise, en particulier à l'étranger. Nous étudierons la diversification sectorielle de la clientèle, c'est-à-dire si l'entreprise possède des clients dans différents secteurs d'activité.

Quatrième hypothèse: Il existe une relation entre la diversification clientèle et l'implication à l'export des PME/PMI.

\subsection{La productivité}

En plus de l'innovation, il nous a paru intéressant d'étudier l'influence de l'utilisation des potentiels techniques et humains des entreprises à travers les employés (productivité) et les équipements, suivant leur part dans la réalisation du chiffre d'affaires.

Cinquième hypothèse: Il existe une relation entre la productivité et l'implication à l'export des PME/PMI.

\subsection{La taille}

Comme le souligne Miesenbock (1988), la taille de l'entreprise est une des variables les plus étudiées dans les recherches sur l'exportation avec des résultats très différents. Cette étude ayant la prétention d'être utile aux entreprises du secteur étudié et en l'absence d'études antérieures sur les soustraitants de l'aéronautique, il semble intéressant de caractériser dans notre cas la relation existant entre la taille et l'exportation. Dans la littérature, la taille est étudiée en prenant comme mesure soit le chiffre d'affaires, soit l'effectif. Nous avons choisi de retenir les deux mesures. Les études étant contradictoires (Miesenbock, 1988) et aucune généralisation n'étant possible (Reid, 1982; Gemunden, 1991), l'influence de la taille dans le cas des sous-traitants sera étudiée, ne sachant pas si elle existe ni dans quel sens elle opère.

Sixième hypothèse: Il existe une relation entre la taille et l'implication à l'export des PME/PMI.

\section{Méthodologie de la recherche et mesures}

\subsection{Les variables de recherche et leur mesure opérationnelle}

* Variable dépendante : l'implication à l'export.

Nous retiendrons l'implication à l'export, ratio dérivé de celui utilisé par Hirsch (1971) et repris par Ong et Pearson (1982). Ce ratio, que l'on peut qualifier de moyenne dynamique, nous semble plus représentatif des performances à l'export que le pourcentage du CA vendu à l'étranger. 
Implication à l'export $=\frac{\sum_{t=1}^{5} t k \frac{\mathrm{E}_{t} * k}{\mathrm{~S}_{t} * k}}{\sum_{t=1}^{5} t k}$

avec: $\mathrm{E}_{t}=$ ventes à l'export pendant la période $t$

$\mathrm{S}_{t}=$ chiffre d'affaires pendant la période $t$

$k=0$ si les données ne sont pas disponibles, sinon,

$k=1$

Les autres variables de recherche et leur mesure opérationnelle sont présentées dans le tableau 1 :

Pour chacune des variables, sauf les deux variables de diversification, les données sont disponibles pour les cinq dernières années. Une valeur moyenne a donc été retenue afin de renforcer les caractéristiques structurelles des variables employées et d'atténuer les éventuels écarts conjoncturels sur une année.

\section{2. Échantillon}

Nous avons retenu l'ensemble de la population des sous-traitants de l'industrie aérospatiale tel qu'il est communiqué par la Chambre régionale de commerce et d'industrie (CRCI) de la région Midi-Pyrénées, soit 181 entreprises.

\subsection{Collecte des données et instruments de recherche}

Les données utilisées proviennent de deux sources secondaires. La première source secondaire est le fichier de la CRCI pour les données de diversification commerciale et technique. La deuxième source secondaire est le fichier d'informations économiques et financières DIANE pour les autres variables. Ces deux sources nous ont permis d'obtenir l'ensemble des variables pour 48 entreprises parmi les 181 , soit $27 \%$ de la population.

\subsection{Traitements statistiques}

Une approche empirique se caractérise par une démarche inductive de tentative d'explication des faits observés, des données recueillies. Ces relations peuvent être déterminées par plusieurs méthodes, et l'outil statistique nous offre 
TABleau 1

Les variables indépendantes et leur opérationnalisation

\begin{tabular}{lll}
\hline $\begin{array}{l}\text { VARIABles } \\
\text { INDÉPENDANTES }\end{array}$ & MESURE & Signification \\
\hline $\begin{array}{l}\text { Diversification } \\
\text { commerciale }\end{array}$ & Nombre de secteurs clients & $\begin{array}{l}\text { Nomenclature employée par la Chambre régionale de commerce et } \\
\text { d'industrie qui retient } 70 \text { secteurs possibles. }\end{array}$ \\
\hline $\begin{array}{l}\text { Diversification } \\
\text { savoir-faire }\end{array}$ & Nombre de savoir-faire & $\begin{array}{l}\text { Ils délimitent les champs de compétences de l'entreprise. Selon la } \\
\text { nomenclature du secteur (développée par la CRCI Midi-Pyrénées), ils se } \\
\text { répartissent dans huit familles principales. La nomenclature de la CRCI } \\
\text { comprend 174 savoir-faire différents. Le nombre de savoir-faire a été } \\
\text { retenu, une catégorisation de type Rumelt (1982) ne nous paraissant pas } \\
\text { opérationnelle pour les PME /PMI. }\end{array}$ \\
\hline
\end{tabular}

Variables

Comme pour plusieurs études antérieures (Cool et Schendel, 1987, 1988; Johnson et Thomas, 1988 ; Lewis et Thomas, 1990), les données comptables sont les sources initiales de ces mesures. Lewis et Thomas (1990) expliquent qu'il existe de multiples liens entre la stratégie et la perfor-

mance. Il est donc souhaitable d'employer plusieurs mesures de performance.

\begin{tabular}{|c|c|c|c|c|}
\hline Endettement ${ }^{1}(\%)$ & $\frac{\text { Dettes financières }}{\text { Ressources durables }}$ & $x$ & 100 & $\begin{array}{l}\text { C'est la part des dettes à caractère financier dans les ressources } \\
\text { durables. C'est une mesure de structure de l'entreprise. }\end{array}$ \\
\hline $\begin{array}{l}\text { Rentabilité } \\
\text { économique }^{2}(\%)\end{array}$ & $\begin{array}{l}\text { Excédent brut d'exploitation } \\
\text { Chiffre d'affaires net }+ \\
\text { Subventions d'exploitation }\end{array}$ & $x$ & 100 & $\begin{array}{l}\text { Mesure de la rentabilité de l'entreprise, qui neutralise l'incidence } \\
\text { des politiques d'amortissements et des politiques financières. }\end{array}$ \\
\hline $\begin{array}{l}\text { Bénéfice net après } \\
\text { impôts }(\%)\end{array}$ & $\begin{array}{l}\text { Bénéfice net après impôts } \\
\text { Chiffre d'affaires }+ \\
\text { Subventions d'exploitation }\end{array}$ & $x$ & 100 & $\begin{array}{l}\text { Mesure de la rentabilité nette de l'entreprise. Cette mesure } \\
\text { représente la part du chiffre d'affaires qui reste dans l'entreprise } \\
\text { (marge). }\end{array}$ \\
\hline $\begin{array}{l}\text { Capacité } \\
\text { d'autofinancement }{ }^{3} \\
(\%)\end{array}$ & $\begin{array}{l}\text { Capacité d'autofinancement } \\
\text { avant répartition } \\
\text { Chiffre d'affaires net }+ \\
\text { Subventions d'exploitation }\end{array}$ & $x$ & 100 & $\begin{array}{l}\text { Cela correspond à la part du chiffre d'affaires qui est disponible pour le } \\
\text { financement propre de l'entreprise (surplus monétaire de l'entreprise). }\end{array}$ \\
\hline
\end{tabular}

1. Les ressources durables sont constituées par : capitaux propres + autres fonds propres + provisions pour risques et charges + dettes à caractère financier + écarts de conversion passif + amortissements et provisions - capital souscrit non appelé - prime de remboursement des obligations.

2. L'excédent brut d'exploitation est ce qu'il reste à l'entreprise après avoir enlevé de la valeur ajoutée créée par l'entreprise, les charges de personnel et les impôts et taxes, et après avoir rajouté les subventions d'exploitation.

3. Elle correspond à la marge brute d'autofinancement moins les dividendes au titre de l'exercice. 
TABleau 1 (suite)

Les variables indépendantes et leur opérationnalisation

\begin{tabular}{|c|c|c|}
\hline \multicolumn{3}{|l|}{$\begin{array}{l}\text { Variables de } \\
\text { productivité }\end{array}$} \\
\hline $\begin{array}{l}\text { Chiffre d'affaires / } \\
\text { effectif }\end{array}$ & Mesuré en KF par emploi & $\begin{array}{l}\text { Mesure la capacité de l'effectif permanent moyen à dégager du chiffre } \\
\text { d'affaires. }\end{array}$ \\
\hline $\begin{array}{l}\text { Immobilisations } \\
\text { nettes en } \\
\text { installations } \\
\text { techniques, } \\
\text { matériels et } \\
\text { outillages }\end{array}$ & En pourcentage du chiffre d'affaires & $\begin{array}{l}\text { Cette variable renforce la précédente en montrant ce que les } \\
\text { immobilisations en machines génèrent en termes de chiffre d'affaires. } \\
\text { C'est un coefficient d'efficacité. Le fait de prendre une valeur nette peut } \\
\text { modifier ce ratio, car il prend en compte la politique d'amortissement. } \\
\text { Cependant, comme il est retenu une valeur moyenne sur } 5 \text { ans, on obtient } \\
\text { un lissage de cet effet. }\end{array}$ \\
\hline \multicolumn{3}{|l|}{$\begin{array}{l}\text { Variables } \\
\text { d'innovation }\end{array}$} \\
\hline $\begin{array}{l}\text { Frais de recherche } \\
\text { et développements nets }\end{array}$ & En \% du CA & Mesure classique de l'innovation (Morbey, 1988, Lefebvre et al., 1992). \\
\hline $\begin{array}{l}\text { Concessions, } \\
\text { brevets et droits } \\
\text { similaires nets }\end{array}$ & En \% du CA & $\begin{array}{l}\text { Ce critère permet également de vérifier l'activisme de l'entreprise en } \\
\text { matière d'innovation et complète la variable précédente. En effet, la } \\
\text { technologie pouvant s'acquérir directement (Frais de R-D) ou par achat } \\
\text { (Brevets...), cette variable caractérise donc également l'activité } \\
\text { innovatrice de la firme (Kamien et Schwartz, 1982). }\end{array}$ \\
\hline
\end{tabular}

Variables

de taille

Chiffre d'affaires

$\mathrm{KF}$

Variable classique utilisée, entre autres, par Khandwalla (1974).

moyen

Effectif moyen

Mesure classique de la taille ${ }^{4}$ (Miesenbock, 1988).

4. Voir pour ces deux variables, Gooding et Wagner, 1985 
l'avantage de proposer différentes positions pour étudier les relations entre des variables. Afin d'obtenir la vue la plus complète ou plusieurs vues différentes du phénomène étudié et de présenter différentes validations de nos hypothèses, il convient d'exposer différentes analyses statistiques. Conformément à l'objectif de la recherche, nous allons donc tenter de mettre en valeur les variables caractéristiques d'une implication à l'export, c'est-à-dire à la fois le fait d'exporter, mais également le niveau des exportations.

\section{Résultats}

\subsection{Présentation des résultats}

Les variables présentées (tableau 1 de l'annexe 1) caractérisent les PME/PMI de l'échantillon. Ce tableau met en valeur la diversité des petites structures en général et des sous-traitants de l'aéronautique en particulier. La dispersion sur l'ensemble des variables est en effet très importante. En ce qui concerne l'implication à l'export, centre d'intérêt de cette recherche, on constate que cette variable s'étend de 0 à $30 \%$ du chiffre d'affaires, avec une moyenne de $4,71 \%$ et un écart type de 7,21\%. L'étude des corrélations entre les variables offre une première validation de l'hypothèse 6 . L'implication à l'export est en effet positivement corrélée avec la taille $(0,45)$ et de façon significative $(\mathrm{p}<0,01)$. On note donc une première relation entre ces deux variables. Conformément au design de recherche, nous allons en premier lieu tenter de différencier les exportateurs des non-exportateurs.

\subsubsection{Différenciation exportateurs/non-exportateurs}

Un tiers (33\%) des entreprises de notre échantillon n'est pas du tout exportateur. Cela permet d'offrir une première tentative de validation de notre préoccupation présente en étudiant les éventuelles différences significatives sur les moyennes des variables entre les groupes des exportateurs et celui des non-exportateurs (tableau 2).

La lecture de ce tableau souligne le fait que les entreprises exportatrices ont des immobilisations plus importantes en matériels et installations techniques, en pourcentage du chiffre d'affaires, que les non exportatrices. Ce résultat peut s'interpréter de deux façons: soit les exportateurs disposent effectivement de plus d'équipements que les non-exportateurs, soit ces immobilisations génèrent moins de chiffre d'affaires, ce qui traduirait un suréquipement ou une sousutilisation des moyens. Le deuxième résultat est également intéressant. Les exportateurs sont moins diversifiés que les non-exportateurs avec 5,15 secteurs clients en moyenne pour les premiers (avec un écart type de 1,6) et 5,9 pour les seconds (avec un écart type de 1,4). Si ce nombre de secteurs clients peut 
TABleau 2

Caractéristiques des entreprises selon l'exportation

\begin{tabular}{lccc}
\hline Variables & Entreprises & Entreprises & $\mathbf{p}^{\mathbf{1}}$ \\
\hline Effectif moyen & 52,8 & 61,7 & 0,323 \\
Rentabilité économique & 7,73 & 8,83 & 0,330 \\
Capacité d'autofinancement & 4,64 & 4,57 & 0,489 \\
Chiffre d'affaires / effectif & 507,3 & 548,6 & 0,297 \\
Endettement & 24,8 & 28,4 & 0,261 \\
Bénéfice moyen & 2,98 & 2,56 & 0,401 \\
Chiffre d'affaires moyen & 22971 & 29353 & 0,230 \\
Immobilisations, & & & \\
installations techniques, & 5,12 & 8,52 & 0,097 \\
(\% CA) & & & \\
Immobiliations, brevets & 0,02 & 0,30 & 0,118 \\
et concessions & 0,0003 & 0,218 & 0,214 \\
Immobilisations R-D & 5,46 & 5,42 & 0,469 \\
Diversification savoir-faire & 5,93 & 5,15 & 0,055 \\
Diversification clients & & & \\
\hline
\end{tabular}

1 : Test unilatéral.

paraître important, il montre tout de même que la diversification ne semble pas faire partie des atouts pour une firme qui souhaite exporter. Cela supporte l'hypothèse 2 de l'existence d'une relation entre diversification clients et implication à l'export.

Ce résultat peut également être étayé par une analyse discriminante des entreprises exportatrices et non exportatrices (tableau 3). Afin d'éviter des problèmes de multicolinéarité, les variables fortement corrélées ont été éliminées (coefficient de corrélation $>0,5$ ou $<-0,5$; règle suggérée par Bails et Peppers (1982) (voir tableau 1 de l'annexe 1).

Cette analyse montre, d'une part, la pertinence des variables étudiées puisqu'elles permettent de classer correctement $71 \%$ des entreprises et, d'autre part, l'importance de la diversification clients. En effet, cette variable est la plus discriminante dans l'implication export $(-0,89)$ et de signe négatif. La diversification savoir-faire est également discriminante (troisième coefficient). Les autres résultats sont cependant nouveaux. En effet, les variables de productivité sont discriminantes dans l'implication à l'export (deuxième et quatrième coefficients) alors qu'elles n'apparaissaient pas dans les analyses précédentes. De plus, la taille n'intervient qu'en cinquième position. Enfin, le pouvoir discriminant de la $R-D$ est très faible (coefficient $=0,089$ ). Nous allons donc pousser l'analyse plus loin en essayant de différencier les entreprises selon le niveau de leur implication à l'export. 
TABleau 3

Analyse discriminante - Méthode directe

\section{Variables étudiées :}

- une variable de taille: chiffre d'affaires

- deux variables de diversification : clients et savoir-faire

- une variable d'innovation: R-D en \% du CA

- deux variables financières : rentabilité économique et endettement

- deux variables de productivité : chiffre d'affaires/effectif et immobilisations en matériels et installations techniques.

Résultats de la classification :

Appartenance prédictive

$\begin{array}{llcc}\text { Groupe actuel } & & & \\ & & \text { Non-exportateur } & \text { Exportateur } \\ & \text { Non-exportateur } & 80,0 \% & 20,0 \% \\ & \text { Exportateur } & 33,3 \% & 66,7 \%\end{array}$

Pourcentage d'entreprises correctement classées : 70,83\%

Autres résultats :

Valeur propre $=0,1565$, corrélation canonique $=0,3679$; Wilks'Lambda $=0,864$

Box's $M=0,084$ avec un niveau de signification de 0,773

(test de l'égalité de covariance des fonctions canoniques discriminantes)

Coefficients de la fonction discriminante (canoniques standardisés) par ordre d'importance :

- Diversification clients : $-0,893$

- Immobilisations en installations techniques : 0,484

- Diversification savoir-faire : 0,472

- Chiffre d'affaires/effectif: 0,345

- Chiffre d'affaires : 0,334

- Endettement : 0,23929

- R-D : 0,089

- Rentabilité économique : $-0,074$

\subsubsection{Caractérisation de l'importance de l'implication export}

Étant donnée la distribution des sous-traitants selon leur implication export (moyenne à $4,71 \%$, mais implication médiane à $2,01 \%$ ), il semble également intéressant d'étudier le profil des entreprises qui n'exportent pas ou peu par rapport à celui des exportateurs plus importants (tableau 4). 
TABleau 4

Caractéristiques des entreprises suivant le niveau d'implication export

\begin{tabular}{lccr}
\hline Variables & $\begin{array}{c}\text { Export inférieur à la } \\
\text { médiane (2,01 \%) }\end{array}$ & $\begin{array}{c}\text { Export supérieur à la } \\
\text { médiane } \mathbf{( 2 , 0 1 ~ \% ) ~}\end{array}$ & $\mathbf{p}^{\mathbf{1}}$ \\
\hline Effectif moyen & 44,51 & 73,88 & 0,005 \\
Rentabilité économique & 7,25 & 9,72 & 0,143 \\
Capacité d'autofinancement & 4,02 & 5,16 & 0,324 \\
Chiffre d'affaires / effectif & 551,8 & 519,6 & 0,327 \\
Endettement & 26,8 & 27,7 & 0,432 \\
Bénéfice moyen & 2,16 & 3,22 & 0,252 \\
Chiffre d'affaires moyen & 20777 & 33941 & 0,048 \\
Immobilisations, & & & \\
installations techniques & 6,49 & 8,42 & 0,214 \\
Immobilisations, brevets & & & \\
et concessions & 0,03 & 0,40 & 0,0445 \\
Immobilisations R-D & 0,0002 & 0,300 & 0,118 \\
Diversification savoir-faire & 5,29 & 5,58 & 0,282 \\
Diversification clients & 5,62 & 5,16 & 0,158 \\
\hline
\end{tabular}

$1:$ Test unilatéral.

L'analyse de ce tableau montre des résultats sensiblement différents des précédents. On remarque que les entreprises plus fortement exportatrices ont une taille globale plus importante avec un effectif moyen de 74 employés contre 45 et un chiffre d'affaires moyen de $34 \mathrm{MF}$ contre $21 \mathrm{MF}$. L'effet de taille semble donc important pour l'implication à l'export puisque les entreprises faiblement ou non exportatrices peuvent se classer dans la catégorie des petites entreprises (effectif $<50$ employés) et les plus fortement exportatrices dans la catégorie des moyennes entreprises. C'est là une première indication intéressante quant aux atouts à maîtriser pour exporter durablement. Ce résultat conforte donc la sixième hypothèse. La deuxième remarque concerne la politique d'investissement en R-D de la firme. On constate en effet des différences significatives dans la part du chiffre d'affaires consacrée aux immobilisations en brevets et concessions $(p<0,05)$ et en immobilisations en recherche et développement $(p \approx 0,1)$. L'acquisition ou la maîtrise d'un savoir-faire particulier, plutôt par achat de savoir-faire (brevets et concessions) qu'en interne (frais de R-D) semble déterminante dans le cadre d'une politique d'internationalisation des sous-traitants. Il existerait donc une relation entre l'innovation et l'implication à l'export, comme le propose la première hypothèse.

Pour compléter cette analyse, il semble pertinent d'étudier dans quelle mesure les différentes variables permettent d'expliquer le degré d'implication à l'export. Cependant, étant donné le nombre de variables étudiées par rapport 
à la taille de l'échantillon et aux corrélations entre certaines d'entre elles (tableau 1, annexe 1), nous avons souhaité tout d'abord les regrouper afin de déterminer les pôles d'étude de l'internationalisation des PME/PMI. L'analyse factorielle avec rotation varimax a été choisie, car elle nous assure une absence de multicolinéarité, une des hypothèses de base de la régression multiple. Les résultats sur les variables précédentes mettent en valeur cinq facteurs très différents (tableau 5).

TABLEAU 5

Résultats de l'analyse factorielle avec rotation varimax

\begin{tabular}{|c|c|c|c|c|c|}
\hline Variables indépendantes & Facteur 1 & Facteur 2 & Facteur 3 & Facteur 4 & Facteur 5 \\
\hline \multicolumn{6}{|l|}{ Variables financières: } \\
\hline Capacité d'autofinancement & 0,95 & 0,09 & $-0,01$ & 0,002 & 0,11 \\
\hline Bénéfice moyen & $\mathbf{0 , 9 2}$ & 0,10 & $-0,08$ & 0,002 & $-0,11$ \\
\hline Rentabilité économique & $\mathbf{0 , 9 2}$ & 0,13 & $-0,03$ & $-0,09$ & 0,25 \\
\hline Endettement & $-0,59$ & 0,54 & $-0,02$ & 0,08 & 0,33 \\
\hline $\begin{array}{l}\text { Variables d'innovation : } \\
\text { Immobilisations en R-D }\end{array}$ & 0,12 & 0,95 & 0,01 & 0,06 & $-0,02$ \\
\hline $\begin{array}{l}\text { Immobilisations en brevets } \\
\text { et concessions }\end{array}$ & 0,14 & 0,95 & 0,05 & $-0,10$ & 0,07 \\
\hline \multicolumn{6}{|l|}{ Variables de taille: } \\
\hline Chiffre d'affaires & $-0,04$ & 0,06 & 0,96 & 0,08 & $-0,19$ \\
\hline Effectif & $-0,05$ & $-0,008$ & 0,94 & 0,21 & 0,11 \\
\hline \multicolumn{6}{|l|}{ Variables de diversification: } \\
\hline Savoir-faire & 0,09 & 0,04 & 0,09 & $\mathbf{0 , 8 5}$ & 0,09 \\
\hline Clients & $-0,16$ & $-0,03$ & 0,18 & 0,77 & $-0,05$ \\
\hline \multicolumn{6}{|l|}{ Variables de productivité: } \\
\hline $\mathrm{CA} / \mathrm{effectif}$ & $-0,01$ & 0,08 & 0,20 & $-0,27$ & $-\mathbf{0 , 8 1}$ \\
\hline Immobilisations techniques ( $\% \mathrm{CA}$ ) & 0,16 & 0,28 & 0,19 & $-0,36$ & $\mathbf{0 , 7 3}$ \\
\hline Valeur propre & 3,31 & 2,38 & 2,04 & 1,45 & 1,18 \\
\hline $\begin{array}{l}\text { Pourcentage cumulé } \\
\text { de variance expliquée }\end{array}$ & $27,6 \%$ & $47,5 \%$ & $64,5 \%$ & $76,7 \%$ & $86,6 \%$ \\
\hline
\end{tabular}

Mesure Kaiser-Meyer-Olkin de l'adéquation de l'échantillon : 0,49498.

Le premier facteur, qui explique $27,6 \%$ de la variance expliquée totale, est constitué des variables représentatives de la santé financière de l'entreprise. Le deuxième facteur représente la politique d'innovation de la firme, politique d'acquisition externe (achat de brevets et concessions) et interne (R-D). Le troisième facteur caractérise la taille de l'entreprise. Le quatrième facteur regroupe la diversification de l'entreprise, à travers le nombre de savoir-faire maîtrisés et le nombre de secteurs clients. Enfin, le cinquième facteur rend compte de la productivité de l'entreprise à travers l'utilisation de son équipement et de son personnel. Ces cinq facteurs représentent $86 \%$ de l'ensemble 
de la variance expliquée et donnent cinq dimensions bien spécifiques des entreprises étudiées. Les dimensions de l'analyse factorielle correspondent aux dimensions du cadre conceptuel. Ce résultat est intéressant, car il justifie et valide dans une certaine mesure le choix des variables qui caractérisent les dimensions. Il est opportun de voir comment ces facteurs permettent d'expliquer le niveau d'exportation des entreprises.

Les résultats de la régression multiple par étape sur les facteurs précédents, en prenant comme variable dépendante l'implication à l'export (tableau 6), confirment les résultats précédents. En effet, deux des cinq facteurs entrent dans la régression et permettent d'expliquer pratiquement $30 \%$ de l'implication à l'export.

Tableau 6

Régression multiple par étape sur l'implication à l'export $(n=46)$

\begin{tabular}{lccccc}
\hline $\begin{array}{l}\text { Variables } \\
\text { indépendantes }\end{array}$ & $\begin{array}{c}\mathbf{F} \\
\text { équation de } \\
\text { régression }\end{array}$ & $\mathbf{R}^{2}$ cumulé & $\begin{array}{c}\mathbf{R}^{2} \text { ajusté } \\
\text { cumulé }\end{array}$ & $\begin{array}{c}\text { Coefficients de régression } \\
\text { standardisés }\end{array}$ \\
\hline & & & & lre étape $^{\text {e étape }}$ \\
Facteur ${ }^{\mathrm{a}}$ 3 Taille & $14,16^{* * *}$ & $23,9 \%$ & $22,2 \%$ & $0,49 * * *$ & $0,49 * * *$ \\
$\begin{array}{l}\text { Facteur } \\
\text { Diversification }\end{array}$ & $10,11 * * *$ & $31,5 \%$ & $28,3 \%$ & & $-0,27 *$ \\
\hline
\end{tabular}

$* \mathrm{p}<0,05 \quad * * \mathrm{p}<0,001 \quad * * * \mathrm{p}<0,001$

Durbin-Watson $=2,56$

a Les facteurs sont mesurés par leurs scores factoriels.

L'analyse finale des résidus n'indique pas de violation des hypothèses de base.

Le facteur taille est le facteur le plus important puisqu'il explique $22,2 \%$ de l'export avec un coefficient positif $(\beta=0,49)$. On retrouve ici un très important effet de taille dans l'exportation des entreprises. Exporter dans le cas des sous-traitants de l'aéronautique demande une «taille critique». Cela conforte donc la sixième hypothèse sur la relation entre la taille et l'implication export. Cependant, on ne sait pas si le fait d'atteindre une certaine taille pousse les entreprises à exporter par «saturation» de leur marché domestique ou donne les moyens financiers, humains, en R-D...pour exporter. Le facteur diversification permet d'expliquer $6 \%$ de l'implication export. L'intérêt principal réside dans le fait que l'influence de ce facteur est négative $(\beta=-0,27)$. Les entreprises qui exportent le plus sont donc les moins diversifiées. On peut supposer qu'elles se sont focalisées sur certains marchés et n'ont pas étendu leur domaine de compétence. Ce résultat conforte donc à la fois les hypothèses 2 et 3 . La mise en facteur ne permet cependant pas de montrer si c'est la 
diversification savoir-faire ou la diversification client qui permet d'expliquer l'implication à l'export.

Cependant, la régression effectuée sur les variables permet de répondre à cette précédente question (tableau 7). Nous avons utilisé les mêmes variables que dans l'analyse discriminante (variables fortement corrélées éliminées).

\section{TABleau 7}

Régression multiple par étape sur l'implication à l'export avec les variables de base

\begin{tabular}{lccccc}
\hline $\begin{array}{l}\text { Variables } \\
\text { indépendantes }\end{array}$ & $\begin{array}{c}\text { F } \\
\text { équation de } \\
\text { régression }\end{array}$ & $\mathbf{R}^{2}$ cumulé & $\begin{array}{c}\mathbf{R}^{2} \text { ajusté } \\
\text { cumulé }\end{array}$ & $\begin{array}{c}\text { Coefficients de régression } \\
\text { standardisés }\end{array}$ \\
\hline Chiffre d'affaires & $11,7^{* * *}$ & $20,6 \%$ & $18,9 \%$ & $0,45^{* *}$ & $0,54^{* * *}$ \\
$\begin{array}{l}\text { Nombre } \\
\text { de clients }\end{array}$ & $11,18^{* * *}$ & $33,7 \%$ & $30,7 \%$ & & $-0,37^{* *}$ \\
\hline
\end{tabular}

$* \mathrm{p}<0,05 \quad * * \mathrm{p}<0,001 \quad * * * \mathrm{p}<0,001$

Durbin-Watson $=2,51$

Les hypothèses de base pour conduire la régression sont présentes : les variables indépendantes ne sont pas fortement corrélées ; l'analyse finale des résidus n'indique pas de violation des hypothèses de base.

Si l'on remarque toujours que la taille, à travers le chiffre d'affaires (variable très corrélée avec l'effectif) explique le plus l'export $(18,9 \%)(\beta=0,54)$, la seconde variable qui entre dans la régression est la diversification clients. Cette deuxième variable permet un gain de $11,8 \%$ dans l'explication de l'implication à l'export. Le coefficient de régression est, bien entendu, toujours négatif $(\beta=-0,37)$. On peut donc dire que les entreprises qui exportent le plus sont celles qui sont le moins diversifiées en termes de secteurs clients, ce qui permet d'accepter l'hypothèse 2 de relation entre la diversification clients et l'implication à l'export.

\subsection{Interprétation générale des résultats}

À la lumière des différentes analyses statistiques, il est possible de dégager trois résultats principaux concernant nos hypothèses de départ.

Il existe une relation entre la taille de l'entreprise et l'implication à l'export des sous-traitants de l'aéronautique civile en Midi-Pyrénées. Le chiffre d'affaires est, en effet, la meilleure variable prédictive du niveau d'implication à l'export. Si l'analyse discriminante ne permet pas de dire que la probabilité de devenir exportateur s'accroît avec la taille, on peut cependant 
dire qu'il y a une relation positive entre la taille de l'entreprise et son implication à l'export. Si l'on s'appuie sur la revue de littérature de Miesenbock (1988), ce résultat est dans la tendance des recherches qui montrent que «plus l'entreprise est grande, plus elle commence à exporter facilement et plus elle fait des affaires sur le plan international » (Miesenbock, 1988; 46) (traduction libre). Ainsi, de nombreuses études montrent une relation positive entre le chiffre d'affaires et l'implication à l'export (par exemple, Cavusgil et al., 1979 ; Malekzadeh et Nahavandi, 1985 ; Yaprak, 1985). Cette relation peut cependant s'appuyer sur trois arguments (Bonaccorsi, 1992). Le premier concerne la théorie des ressources limitées qui suppose qu'une faible taille ne permet pas de disposer de ressources suffisantes pour envisager l'exportation sereinement: absence de moyens financiers pour aller sur les marchés directement ou pour créer une représentation commerciale, absence de moyens marketing pour pénétrer les marchés et absence de ressources humaines, notamment de personnel qualifié pratiquant les langues et les cultures étrangères. Cependant, cela est tempéré par le fait que la relation taille/export est dépendante de la stratégie export du dirigeant. Le deuxième argument concerne les économies d'échelle qui peuvent être réalisées en produisant à l'étranger ou en termes de marketing, à l'export. Le troisième argument concerne la perception du risque que le dirigeant peut avoir face à l'exportation. Cet ensemble de problèmes fait que l'exportation n'est pas un phénomène naturel pour les petites entreprises. Dans notre cas, nous nous trouvons en présence de sous-traitants de l'aéronautique, habitués à disposer d'une clientèle présente à moins de $200 \mathrm{~km}$ de leur implantation. Cette clientèle assurait traditionnellement la presque totalité de leur activité. L'intérêt pour l'exportation n'était donc pas fort, car la croissance était régulière et le risque de défaillance de ces clients semblait très faible. Le lien présent entre la taille et le niveau d'exportation n'est pas explicable par les questions financières. En effet, on ne trouve aucun lien entre la performance financière et l'exportation, ni entre la taille et la performance financière. Cependant, cette relation pourrait se justifier par le fait que les sous-traitants se trouvent dans un environnement de volume. Cela expliquerait l'existence d'économies d'échelle et la focalisation nécessaire sur un petit nombre de secteurs clients (suggestion d'un des lecteurs anonymes). Au cours de nos rencontres auprès de plusieurs sous-traitants, nous avons remarqué, mais cela n'est pas vérifié par la présente analyse, que certains sous-traitants parmi les plus importants sont non seulement capables de traiter des quantités plus importantes, mais qu'ils travaillent des pièces plus complexes que les plus petits d'entre eux. Ils disposent donc d'une certaine maîtrise, d'un savoir-faire ou d'une spécialisation utiles dans le cadre de l'exportation. On pourrait dire qu'ils font de la sous-traitance de capacité spécialisée. 
Le deuxième résultat concerne la diversification en termes de secteurs clients. L'ensemble des analyses converge vers le fait que la diversification clients est une variable fortement prédictive de l'implication à l'export des entreprises. Le fait le plus remarquable est que son effet est négatif : ce sont les entreprises les moins diversifiées qui exportent le plus. On peut donc se demander si certains sous-traitants de l'aéronautique ne suivent pas une séquence d'internationalisation (Saporta, 1993). En effet, la présence d'unités de grande dimension dans le secteur pousse les entreprises à une stratégie de spécialisation (niche de clientèle) et de sous-traitance. Mais cette spécialisation entraîne certaines difficultés, notamment une limite à la croissance et une vulnérabilité due à la faible taille relative du marché. Ces risques pousseraient alors les entreprises vers une stratégie de développement international. Cependant, si les résultats semblent conforter ce processus d'internationalisation des entreprises, on peut se demander si la spécialisation, dans le cas des sous-traitants de l'aéronautique, est un atout directement responsable de l'exportation, une faiblesse pour le marché interne qui force les entreprises à vendre à l'étranger ou un mélange des deux phénomènes. En effet, une analyse sur le terrain de plusieurs sous-traitants de ce secteur nous permet de dire que la majorité sont des sous-traitants de capacité, opérant en tôlerie ou en mécanique et ne disposant donc pas d'un réel savoir-faire. La maîtrise d'un ou plusieurs savoir-faire n'est d'ailleurs pas un critère de détermination de l'implication à l'export. De plus, le lien entre le nombre de savoir-faire maîtrisés et le nombre de secteurs clients est significatif, mais faible (voir annexe 1). Il ne faut pas oublier que l'on se trouve en présence de petites unités, souvent dirigées par un seul individu. L'analyse, dégageant des résultats généraux, nous permet de dire que l'exportation ne relève pas que du hasard, de la volonté du dirigeant ou d'une opportunité d'affaires subite, sans que l'on puisse pour autant rationaliser le processus.

De plus, les autres résultats de l'analyse nous permettent de dire que l'exportation n'est pas dépendante de la santé financière ou de la productivité. Cela conforte le fait que la «stratégie d'internationalisation» suivie par les sous-traitants étudiés ne suit pas une démarche unique. Les entreprises exportent, en effet, sans disposer d'une assise financière permettant de soutenir cette décision. Il en est de même concernant la politique en matière d'innovation. En effet, les résultats ne nous permettent pas de conclure. Si les entreprises plus fortement exportatrices ont une politique d'innovation plus importante (moyenne des investissements en R-D et en brevets et concessions plus importantes), ce critère n'est pas prédictif du niveau d'engagement à l'export des entreprises. 


\subsection{Implications pour les entreprises}

Si l'on considère que l'internationalisation est une voie possible pour aider les sous-traitants de l'aéronautique à sortir de leur situation de dépendance aux grands donneurs d'ordre, la constitution d'une taille critique et la focalisation sur certains secteurs industriels sont des atouts dont les entreprises doivent disposer.

Pour obtenir une certaine taille, il convient de favoriser un regroupement des entreprises par la création d'alliances ou de réseaux. Les alliances seront de nature différente suivant les problèmes rencontrés par les PME dans le cadre de leur stratégie d'internationalisation (Forrest, 1990). Ces alliances pourront porter sur le marketing et la distribution afin de créer une structure de force de vente commune utilisable pour défricher les marchés étrangers ou sur la production pour développer les capacités et ainsi être en mesure de répondre aux appels d'offre importants ou à délai de réalisation rapide. Les problèmes de taille relatifs à l'exportation peuvent également être résolus grâce au portage, où la PME portée utilise le réseau international d'une société porteuse (Wissler, 1993). Cependant, dans notre cas, compte tenu des relations actuelles entre les sous-traitants et les donneurs d'ordre (voir l'introduction), il faudrait une modification des mentalités des deux parties pour que ce système puisse se mettre en place convenablement. Cette taille peut enfin s'obtenir par la constitution de réseaux qui s'organisent selon deux logiques (Fourcade, 1993): une logique de valorisation qui consiste à développer une activité réticulaire (qui a la forme d'un réseau) pour réduire les coûts et faire jouer des effets de synergie et une logique d'intention qui utilise la dynamique du réseau pour produire des synergies dans le but de créer de la valeur ajoutée à partir d'un capital relationnel commun aux acteurs. Dans ce cadre, il semble important de privilégier le capital relationnel indispensable à la réticulation. Les quelques exemples de réseaux en formation dans la région montrent clairement que dans le cadre des petites entreprises, les relations humaines sont la clé de voûte de la pérennité du réseau. Le réseau le plus simple à mettre en place actuellement semble être un réseau constitué de sous-traitants de même niveau, car ce sont, d'après nos rencontres avec certaines de ces entreprises, les seuls aptes à s'entendre.

Dans le domaine de la focalisation sectorielle des entreprises, il semble difficile en temps de crise de suggérer aux entreprises de se limiter à un ou deux secteurs, même si cela favorise leur internationalisation. En effet, cellesci sont actuellement dans une phase de combat pour leur survie. Tous les marchés sont bons à conquérir. Un effort de sensibilisation est donc nécessaire. 


\section{Limites de la recherche}

Cette recherche, s'inscrivant dans une démarche empirique et exploratoire, comporte certaines limites en termes de validité externe, compte tenu de la nature et de la taille de l'échantillon. En effet, nous nous sommes intéressés exclusivement aux sous-traitants de l'industrie aéronautique de la région MidiPyrénées. Cette restriction sectorielle et géographique est intéressante dans la mesure où elle permet d'éviter certains biais relatifs à l'influence de l'environnement (Boyer, 1993). Cependant, si la taille de l'échantillon (27\% de la population) permet d'analyser convenablement la situation des sous-traitants de Midi-Pyrénées, elle ne permet pas de dégager des conclusions pour l'ensemble du secteur aéronautique français, ni bien entendu pour les PME françaises en général. Mais ce type de généralisation sort du cadre de notre recherche.

La deuxième limite concerne le choix des variables. Comme dans toute recherche, on peut reprocher à l'auteur d'avoir choisi certaines variables au détriment d'autres. Notre limitation dépend, comme pour de nombreuses recherches, des limites de nos sources secondaires, qui rendent peut-être la recherche moins exhaustive, mais qui ont l'avantage d'être fiables.

\section{Conclusion}

\section{Implications et directions pour une recherche future}

Cette étude, réalisée à partir de données secondaires sur 48 PME/PMI soustraitantes de l'industrie aéronautique, avait pour objectif de mettre en valeur les relations entre l'implication à l'export et différentes variables caractéristiques de la structure des entreprises: taille, performance financière, productivité, diversification en termes de savoir-faire et de clientèle et innovation. Indépendamment des limites citées, cette recherche a permis de faire ressortir les relations entre l'exportation des PME/PMI, la taille et la diversification de la clientèle. Si les préoccupations de ces entreprises sont orientées vers leur internationalisation, elles doivent savoir que celles qui réussissent à exporter durablement sont plutôt des moyennes entreprises que des petites et que ce sont des entreprises qui ont axé leurs débouchés commerciaux sur un nombre restreint de secteurs.

L'atteinte d'une certaine taille et la focalisation sont des atouts à l'internationalisation. Des efforts de recentrage des activités commerciales et de regroupement d'entreprises doivent donc être réalisés dans ce cas. Cependant, cette réponse n'est pas entièrement satisfaisante. Dans le cadre d'une recherche future, il serait judicieux de prolonger ce travail selon deux axes. Le 
premier intérêt réside dans l'extension des variables prises en compte aux caractéristiques de l'environnement et du dirigeant afin de disposer d'un modèle plus général de l'internationalisation des PME/PMI. La deuxième extension de cette recherche devrait, quant à elle, porter sur une comparaison des différentes pratiques d'internationalisation dans les autres pays disposant d'un secteur de PME/PMI sous-traitantes comparable à la région MidiPyrénées afin d'essayer de dégager des pratiques générales dans ce domaine. La présente recherche ne constitue donc qu'une première tentative de détermination de ce phénomène. 
AnNeXe 1

Statistiques descriptives et corrélations $(n=48)$

\begin{tabular}{|c|c|c|c|c|c|c|c|c|c|c|c|c|c|c|c|c|}
\hline & Variables & Moyenne & $\begin{array}{c}\text { e Écart } \\
\text { type }\end{array}$ & V1 & V2 & V3 & V4 & V5 & V6 & V7 & V8 & V9 & V10 & V11 & V12 & V13 \\
\hline V1 & Effectif & 58,89 & 61,01 & 1,00 & & & & & & & & & & & & \\
\hline v2 & Rentabilité & 8,49 & 7,95 & $-0,09$ & 1,00 & & & & & & & & & & & \\
\hline v3 & $\begin{array}{c}\text { Implication } \\
\text { export }\end{array}$ & 4,71 & 7,21 & $0,37^{*}$ & 0,22 & 1,00 & & & & & & & & & & \\
\hline v4 & CAF & 4,60 & 8,50 & $-0,05$ & $0,95 * *$ & * 0,17 & 1,00 & & & & & & & & & \\
\hline v5 & CA/effectif & 535,72 & 245,78 & $-0,04$ & $-0,11$ & 0,03 & $-0,06$ & 1,00 & & & & & & & & \\
\hline V6 & Endettement & 27,27 & 17,36 & 0,04 & $-0,34 *$ & $-0,10$ & $-0,42 * *$ & $*-0,17$ & 1,00 & & & & & & & \\
\hline v7 & Bénéfice & 2,70 & 5,38 & $-0,12$ & $\mathbf{0 , 8 0} * *$ & * 0,16 & $\mathbf{0 , 8 5 * *}$ & $* 0,007$ & $-0,47 * *$ & * 1,00 & & & & & & \\
\hline v8 & $\begin{array}{c}\text { Chiffre } \\
\text { d'affaires }\end{array}$ & 27359 & 27408 & $0,89 * *$ & $-0,12$ & $0,45^{* *}$ & $*_{-} 0,07$ & $0,31^{*}$ & 0,01 & $-0,06$ & 1,00 & & & & & \\
\hline V9 & $\begin{array}{l}\text { Installations } \\
\text { techniques }\end{array}$ & 7,46 & 8,35 & 0,09 & $0,43 * *$ & 0,23 & 0,26 & $-0,25$ & 0,24 & 0,02 & 0,002 & 1,00 & & & & \\
\hline V10 & $\begin{array}{l}\text { Brevets et } \\
\text { concessions }\end{array}$ & 0,22 & 0,75 & 0,02 & 0,27 & 0,12 & 0,21 & 0,02 & $0,36^{*}$ & 0,20 & 0,08 & $0,40 * *$ & * 1,00 & & & \\
\hline V11 & R-D & 0,15 & 0,87 & 0,04 & 0,19 & 0,02 & 0,19 & $-0,03$ & $0,38 * *$ & $* 0,21$ & 0,07 & 0,19 & $\mathbf{0 , 9 3} * *$ & 1,00 & & \\
\hline V12 & Savoir-faire & 5,44 & 1,72 & 0,25 & 0,02 & $-0,05$ & 0,08 & $-0,20$ & 0,06 & 0,03 & 0,14 & $-0,14$ & $-0,01$ & 0,06 & 1,00 & \\
\hline V13 & Clients & 5,40 & 1,57 & 0,27 & $-0,2$ & $-0,24$ & $-0,13$ & $-0,04$ & 0,13 & $-0,18$ & 0,23 & $-0,19$ & $-0,13$ & $-0,02$ & $0,47 * *$ & 1,00 \\
\hline
\end{tabular}




\section{Bibliographie}

AABY, N.E. et S.F. SLATER (1989), « Management influences on export performance : a review of the empirical literature 1978-88 », International Marketing Review, vol. $6, n^{\circ} 4$, p. 7-26.

ACs, Z.J. et D.B. AudretsCh (1990), Innovation and Small Firms, Cambridge, MA, M.I.T. Press.

BAILS, D.G. et L.C. PEPPERS (1982), Business Fluctuation: Forecasting Techniques and Applications, Englewood Cliffs, N.J., Prentice-Hall.

BARTLetT, C.A. et S. Ghoshal (1987a), «Managing across borders : new strategic requirements », Sloan Management Review, été, p. 7-17.

BARTLETT, C.A. et S. GHoshal (1987b), « Managing across borders : new organizational responses », Sloan Management Review, automne, p. 43-53.

Birley, S. et P. WeSTHEAD (1990), «Growth and performance contrasts between “types" of small firms », Strategic Management Journal, vol. 11, p. 535-557.

BONACCORSI, A. (1992), "On the relationship between firm size and export intensity », Journal of International Business Studies, vol. 23, $\mathrm{n}^{\circ} 4,4^{\mathrm{e}}$ trim., p. 605-636.

BOYER, M. (1993), «La performance des PMI dans l'attaque des marchés internationaux - Application au cas des punchs et liqueurs réunionnais », Actes du colloque PME/PMI, développement international, Aix-en-Provence, juin 1993, p. 121-150.

Burton, F.N. et B.B. SCHLEgelmiLCH (1987), «Profile analyses of non-exporters versus exporters grouped by export involvement », Management International Review, vol. 27, n 1, p. 38-49.

CAlORI, R. et Y. HARVATOPOUlos (1988), «Diversification: les règles de conduites », Harvard-l'Expansion, printemps.

CAvusgil, S.T., W.J. Bilkey et G. Tesar (1979), «A note on the export behaviour of firms : export profiles », Journal of International Business Studies, vol. 10, $\mathrm{n}^{\circ} 1$, p. $91-97$.

COOL, K.O. et D.E. SCHENDEL (1987), «Strategic group formation and performance: the case of the US pharmaceutical industry: 1963-1982», Management Science, vol. 33, $\mathrm{n}^{\circ} 9$, p. 1102-1124.

Cool, K.O. et D.E. SCHENDEL (1988), «Performance differences among strategic group members », Strategic Management Journal, vol. 9, n 3, p. 207-223.

COMITÉ ÉCONOMIQUE ET SOCIAL DE LA RÉGION MIDI-PYRÉNÉES (1990), Rapport sur l'industrie aéronautique civile en Midi-Pyrénées, Conseil régional, Toulouse, juin. 
CZINKota, M.R. et W.J. JOHNSTON (1983), «Exporting: does sales volume make a difference? », Journal of International Business Studies, vol. 14, n 1, p. 147-153.

DAMANPOUR, F. (1991), «Organizational innovation: a meta-analysis of effects of determinants and moderators », Academy of Management Journal, vol. 34, n ${ }^{\circ} 3$, p. 555-590.

Detrie, J.P., V. MERCier et B. RAmanantsoA (1982), « Cinq règles pour se diversifier», Harvard-l'Expansion, hiver 1981-1982, p. 37-55.

Dichtl, E., M. Leibold, H.G. KögmmayR et S. MÜller (1984), « The exportdecision of small and medium sized firms: a review », Management International Review, vol. 24, n² 2, p. 49-60.

FORREST, J.E. (1990), "Strategic alliances and the small technology-based firm », Journal of Small Business Management, juillet, p. 37-45.

FourCade, C. (1993), «Stratégies de réseau: un modèle stratégique de l'internationalisation des petites entreprises », Actes du colloque PME/PMI, développement international, Aix-en-Provence, juin 1993, p. 305-323.

GEMUNDEN, H.G. (1991), «Success factors of export marketing : a meta analytic critique of the empirical studies », dans S.J. Paliwoda, New Perspectives on International Marketing, London, Routledge.

GoODING, R.Z. et J.A. WAGNER (1985), «A meta-analytic review of the relationship between size and performance: the productivity and efficiency of organizations and their subunits », Administrative Science Quarterly, vol. 30, p. 462-481.

JOHNSON, G. et H. ThOMAS (1988), «Strategic groups and financial performance : a critical examination», Cahier de recherche, Manchester Business School.

KAMIEN, M.I. et N.L. SChWARTZ (1982), Market Structure and Innovation, Cambridge, MA, Cambridge University Press.

Khandwalla, P.N. (1977), The Design of Organizations, New York, Harcourt Bruce Jovanovitch Inc.

KAYNAK, E., P.N. Ghauri et T. OlofSSON-BREDEnlöw (1987), «Export behaviour of small Swedish firms », Journal of Small Business Management, vol. 25, $\mathrm{n}^{\circ} 2$, p. 26-32.

KIRPALANI, V.H. et N.B. MACINTOSH (1980), «Internal marketing effectiveness of technology oriented small-firms », Journal of International Business Studies, vol. 11, hiver, p. 81-90.

LefeBVRE, L.A., J. HARVEY et E. LefEBVRE (1991), «Technological experience and the technology adoption decisions in small manufacturing firms », $R-D$ Management, vol. 21, $\mathrm{n}^{\circ} 3$, p. 241-249.

Lefebvre, L.A., A. Langley, J. Harvey et E. Lefebvre (1992), «Exploring the strategy-technology connection in small manufacturing firms ", Production and Operations Management, vol. 1, n 3, p. 269-285. 
Lefebvre, L.A. et E. LeFebVRE (1992), «Firm innovativeness and CEO characteristics in small manufacturing firms », Journal of Engineering and Technology Management, vol. 9, p. 243-277.

LEWIS, P. et H. ThOMAS (1990), "The linkage between strategy, strategic group and performance in the U.K. retail grocery industry », Strategic Management Journal, vol. 11, p. 385-397.

MC Gee, J. et H. Thomas (1989), «Technology and strategic management, progress and future directions », $R$-D Management, vol. 19, $\mathrm{n}^{\circ} 3$, p. 205-213.

MCGuiness, N. et N. LiTTLE (1981), «The influence of product characteristics on the export performance of new industrial products », Journal of Marketing, vol. 45, printemps, p. 110-122.

Malekzadeh, A.R. et A. Nahavandi (1985), "Small business exporting: misconceptions are abundant », American Journal of Small Business, vol. IX, nº 4, printemps, p. 7-14.

MiESENBOCK, K.J. (1988), «Small businesses and exporting : a literature review », International Small Business Journal, vol. 6, $\mathrm{n}^{\circ}$ 2, janvier-mars, p. 42-61.

MORBEY, G.K. (1988), «R-D : Its relationship to company performance », Journal of Production Innovation Management, vol. 5, p. 191-200.

OBSERVATOIRE DE LA SOUS-TRAITANCE DE L'INDUSTRIE AÉRONAUTIQUE ET SPATIALE (OSTIAS) (1992), Synthèse de 30 audits stratégiques de sociétés soustraitantes adhérentes du GIFAS, Paris, GIFAS, décembre.

OGRAM, E.W. (1982), « Exporters and non-exporters : a profile of small manufacturing firms in Georgia ", dans M.R. Czinkota et G. Tesar, Export Management : An International Context, New York, Praeger Publishers, p. 70-84.

Olson, H.C. et F. WiEDERSHEIM-PAUL (1978), «Factors affecting the pre-export behaviour of non-exporting firms », dans M. Ghertman et J. Leontiades (éd.), European Research in International Business, New York, p. 283-305.

ONG, C.H. et A.W. PEARSON (1982), «The impact of technical characteristics on export activity : a study of small and medium-sized UK electronics firms », $R-D$ Management, vol. 12, $\mathrm{n}^{\circ} 4$, p. 189-196.

REID, S.D. (1982), «The impact of size on export behavior in small firms », dans M.R. Czinkota et G. Tesar, Export Management : An International Context, New York, Praeger Publishers, p. 18-38.

Roux, E. (1991), «Les facteurs explicatifs de la décision d'exporter en PMI : rôle de l'attitude du dirigeant envers le risque », Thèse de Doctorat ès sciences de gestion, IAE d'Aix-Marseille III - ESSEC, janvier.

RuMELT, R.P. (1982), «Diversification strategy and profitability », Strategic Management Journal, vol. 3, p. 359-369. 
SAPORTA, B. (1993), «Les enjeux de l'ouverture internationale des PME», Actes du colloque PME/PMI, développement international, Aix-en-Provence, juin 1993, p. 811-826.

SCHLEgelmilCH, B.B. et J.N. CROOK (1986), «Firm level determinants of export intensity », Cahier de recherche 86-9, Université d'Edinburgh.

TOOKEY, D.A. (1964), «Factors associated with success in exporting », Journal of Management Studies, mars, p. 48-66.

Wiedersheim-PAUl, F., H. Olson et L. Welch (1978), «Pre-export activity: the first step in internationalisation », Journal of International Business Studies, vol. $9, \mathrm{n}^{\circ} 1$, p. $47-58$.

WISSLER, M. (1993), «Le portage à l'export et les stratégies relationnelles des PME», Actes du colloque PME/PMI, développement international, Aix-en-Provence, juin 1993, p. 851-867.

YAPRAK, A. (1985), «Empirical study of the differences between small exporting and non exporting US firms », International Marketing Review, vol. 2, p. 72-83. 